\title{
POROSOME: THE UNIVERSAL SECRETORY PORTAL IN CELLS
}

\author{
Bhanu P. Jena \\ Department of Physiology, Wayne State University School of Medicine, Detroit, MI, USA
}

In the past 50 years it was believed that during cell secretion, membrane-bound secretory vesicles completely merge at the cell plasma membrane resulting in the diffusion of intra-vesicular contents to the cell exterior and the compensatory retrieval of the excess membrane by endocytosis. This explanation made no sense or logic, since following cell secretion partially empty vesicles accumulate as demonstrated in electron micrographs. Furthermore, with the 'all or none' mechanism of cell secretion by complete merger of secretory vesicle membrane at the cell plasma membrane, the cell is left with little regulation and control of the amount of content release. Moreover, it makes no sense for mammalian cells to possess such 'all or none' mechanism of cell secretion, when even single-cell organisms have developed specialized and sophisticated secretory machinery, such as the secretion apparatus of Toxoplasma gondii, the contractile vacuoles in paramecium, or the various types of secretory structures in bacteria. Therefore, in the 1960's, experimental data concerning neurotransmitter release mechanisms by B. Katz and B. Folkow brilliantly hypothesized that limitation of the quantal packet may be set by the nerve membrane, in which case the size of the packet may actually correspond to just a fraction of the vesicle content. This conundrum in the molecular mechanism of cell secretion was finally resolved in 1997 following discovery of the porosome, the universal secretory machinery in the cell. Porosomes are supramolecular lipoprotein structures at the cell plasma membrane, where membrane-bound secretory vesicles transiently dock and fuse to release inravesicular contents to the outside during cell secretion. In the past decade, the composition of the porosome, its structure and dynamics at nanometer $(\mathrm{nm})$ resolution and in real time, and its functional reconstitution into artificial lipid membrane, have all been elucidated. Since porosomes in exocrine and neuroendocrine cells measure 100-180 nm, and only 20-45\% increase in porosome diameter is demonstrated following the docking and fusion of secretory vesicles (0.2-1.2 $\mu \mathrm{m}$ in diameter), it is concluded that secretory vesicles "transiently" dock and fuse, rather than completely merge at the base of the porosome complex to release their contents to the outside. In agreement, it has been demonstrated that "secretory granules are recaptured largely intact after stimulated exocytosis in cultured endocrine cells"; that "single synaptic vesicles fuse transiently and successively without loss of identity"; and that "zymogen granule (the secretory vesicle in exocrine pancreas) exocytosis is characterized by long fusion pore openings and preservation of vesicle lipid identity". In this review, the discovery of the porosome, resulting in a paradigm shift in our understanding of cell secretion, is briefly presented. Biomed Rev 2010; 21: 1-15.

Key words: cell secretion, membrane fusion, SNAREs, secretory vesicle, neurotransmission, hormone release

Received 10 December 2010, accepted 19 December 2010.

Correspondence: Bhanu P. Jena, PhD, DSc, (hon. doct. mult.), George E. Palade University Professor Distinguished Professor, Department of Physiology, Wayne State University School of Medicine 5245 Scott Hall, Detroit, MI 48201, USA.

Tel.: 313-577-1532, Fax: 313-993-4177, E-mail: bjena@med.wayne.edu

Web: http://www.med.wayne.edu/physiology/facultyprofile/jena 


\section{INTRODUCTION}

Secretion is a fundamental cellular process as old as life, occurs in all living organisms, from the simple yeast to cells in humans. Secretion is responsible for a variety of physiological activities in living organisms, such as neurotransmission, release of hormones and digestive enzymes. Secretory defects in cells are responsible for a host of debilitating diseases. Since the mid 1950's, it was believed that during cell secretion, secretory vesicles completely merge at the cell plasma membrane resulting in the outward diffusion of intravesicular contents, and the compensatory retrieval of the excess membrane by endocytosis. In contrast, the observation of partially empty vesicles in cells following secretion could not be justified according to the above mechanism. Then in the 1960's, experimental data concerning neurotransmitter release mechanisms by Katz (1) and Folkow et al (2), brilliantly hypothesized that limitation of the quantal packet may be set by the nerve membrane, in which case the size of the packet may actually correspond to just a fraction of the vesicle content (2-4). Then again in a News and Views article in Nature, E. Neher wrote: "It seems terribly wasteful that during the release of hormones and neurotransmitters from a cell, the membrane of a vesicle should merge with the plasma membrane to be retrieved for recycling only seconds or minutes later" (5). The only way of determining the molecular mechanism of cell secretion would have been a direct observation of the secretory process at nanometer $(\mathrm{nm})$ resolution in living cells. This was finally achieved and the conundrum resolved when isolated live pancreatic acinar cells were imaged in near physiological buffer using atomic force microscopy (AFM) at high force (200-300 pN), in 2004 (6). This study demonstrated the size and shape of the secretory vesicles called zymogen granules (ZGs) lying immediately below the apical plasma membrane of the pancreatic acinar cell (Fig. 1). Within 2.5 min of exposure to a physiological secretory stimulus, the majority of ZGs within these cells were swellen (Fig. 1), followed by a decrease in ZG size (Fig. 1) by which time cell secretion was complete (Fig. 1). These studies revealed for the first time in live cells, the intracellular swelling of secretory vesicles following stimulation of secretion and their deflation following partial discharge of vesicular contents (6). No loss of secretory vesicles was observed throughout the experiment, demonstrating transient fusion and partial discharge of vesicular contents during cell secretion.

The other major breakthrough in our understanding of cell

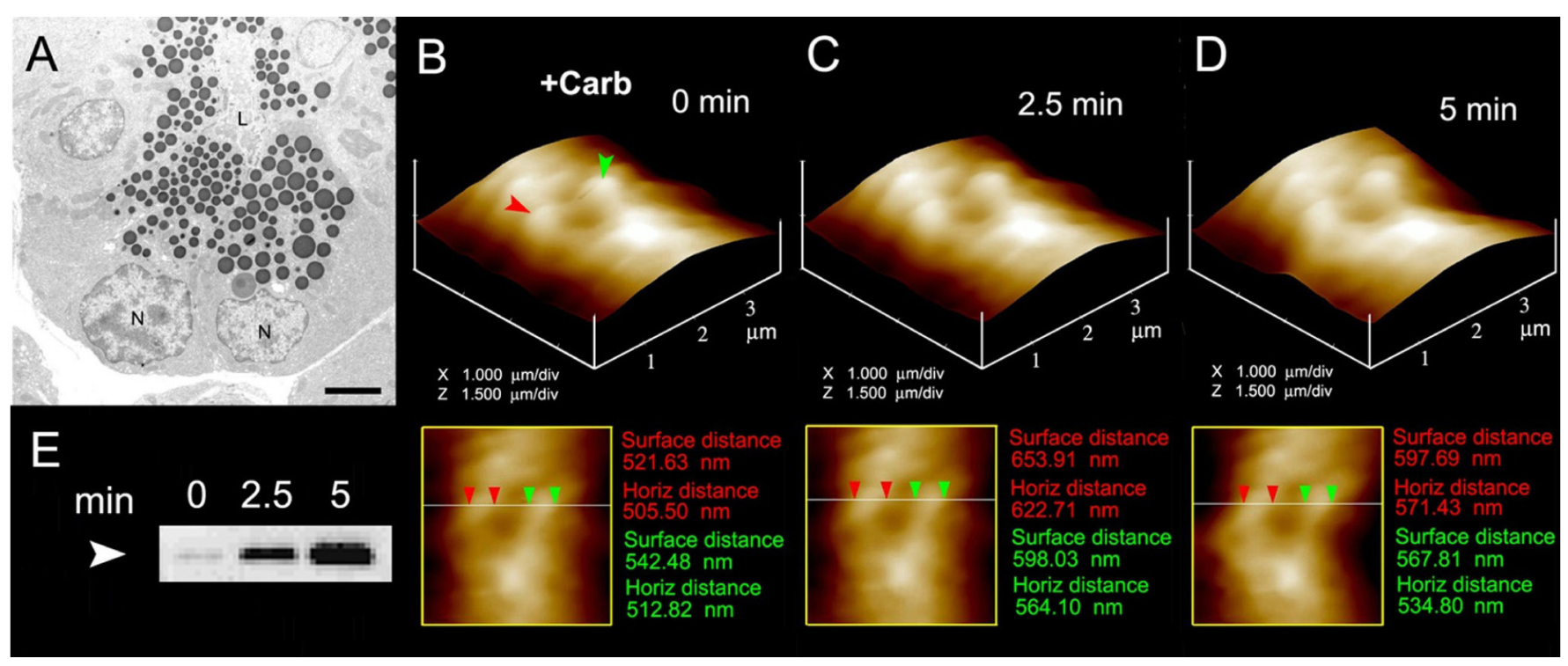

Figure 1. The swelling dynamics of ZGs in live pancreatic acinar cells. (A) Electron micrograph of pancreatic acinar cells showing the basolaterally located nucleus (N) and the apically located ZGs. The apical end of the cell faces the acinar lumen (L). Bar $=2.5 \mu \mathrm{m}$. (B-D) The apical ends of live pancreatic acinar cells were imaged by AFM, showing ZGs (red and green arrowheads) lying just below the apical plasma membrane. Exposure of the cell to a secretory stimulus using $1 \mu M$ carbamylcholine, resulted in ZG swelling within 2.5 min, followed by a decrease in ZG size after 5 min. The decrease in size of ZGs after 5 min is due to the release of secretory products such as a-amylase, as demonstrated by the immunoblot assay (E) (6). 
secretion came with the discovery of a new cellular structure called the 'porosome', using AFM (7-14). In the past decade, the porosome has been determined to be the universal secretory machinery in cells. Porosomes are supramolecular lipoprotein structures at the cell plasma membrane, where membrane-bound secretory vesicles transiently dock and fuse to release inravesicular contents to the outside during cell secretion. The mouth of the porosome opening to the outside, range in size from $150 \mathrm{~nm}$ in diameter in acinar cells of the exocrine pancreas to $12 \mathrm{~nm}$ in neurons, which dilates during cell secretion returning to its resting size following completion of the process. In the past decade, the composition of porosome, its structure and dynamics at $\mathrm{nm}$ resolution and in real time, and its functional reconstitution into artificial lipid membrane, have all been elucidated. Since porosomes in exocrine and neuroendocrine cells measure 100-180 nm, and only $20-35 \%$ increase in porosome diameter is demonstrated following the docking and fusion of secretory vesicles with a diameter of 0.2-1.2 mm, it is concluded that secretory vesicles "transiently" dock and fuse at the base of the porosome complex to release their contents to the outside (Fig. 2). In recent years, using electron microscopy (EM), P. Greengard and associates have identified porosomes-like structures at the presynaptic membrane with docked synaptic vesicles (15). In agreement, other studies have reported that "secretory granules are recaptured largely intact after stimulated exocytosis in cultured endocrine cells" (16), "single synaptic vesicles fusing transiently and successively without loss of identity" (17), and "zymogen granule exocytosis is characterized by long fusion pore openings and preservation of vesicle lipid identity" (18). In nature, even single-cell organisms have developed specialized and sophisticated secretory machinery, such as the secretion apparatus of Toxoplasma gondii (19), the contractile vacuoles in paramecium (20), or the various types of secretory structures in bacteria (21).

\section{POROSOME DISCOVERY}

Porosomes were first discovered in acinar cells of the exocrine pancreas (7). Exocrine pancreatic acinar cells are polarized secretory cells possessing an apical and a basolateral end. This well characterized cells of the exocrine pancreas synthesize digestive enzymes, which are stored within 0.2$1.2 \mu \mathrm{m}$ in diameter apically located secretory vesicles, called zymogen granules (ZGs). Following a secretory stimulus, ZGs dock and fuse with the apical plasma membrane to release their contents to the outside. Contrary to neurons, where secretion of neurotransmitters occurs in the millisecond time regime, the pancreatic acinar cells secrete digestive enzymes over minutes following a secretory stimulus, and are therefore ideal for investigating the molecular steps involved in cell secretion. In the mid 1990's, AFM studies were undertaken on living pancreatic acinar cells to evaluate the structure and dynamics of the apical plasma membrane at high resolution under both resting and stimulated conditions. To our disbelief, isolated live pancreatic acinar cells in physiological buffer when imaged using the AFM revealed new cellular structures. At the apical plasma membrane, a group of circular 'pits' measuring $0.4-1.2 \mu \mathrm{m}$ in diameter containing smaller 'depressions'-like structures were identified (Fig. 2A). Each depression measure between $100-180 \mathrm{~nm}$ in diameter, and 3-4 depressions were typically found within a pit. In contrast, the basolateral membrane of acinar cells was devoid of such structures. High-resolution AFM images of these depressions in live acinar cells further revealed a coneshaped morphology with each cone measuring $15-35 \mathrm{~nm}$ in depth. Subsequent studies over the years demonstrated the presence of such depressions in all secretory cells examined. Analogous to pancreatic acinar cells, examination of resting growth hormone (GH) secreting cells of the pituitary (9) and chromaffin cells of the adrenal medulla (22) also revealed the presence of pits and depressions at the cell plasma membrane. The presence of depressions or porosomes in neurons, astrocytes, $\beta$-cells of the endocrine pancreas and in mast cells have also been determined, demonstrating their universal presence in secretory cells (23-25).

\section{See Editorial on pp 17-24}

Exposure of pancreatic acinar cells to a secretagogue results in a time-dependent increase $(20-45 \%)$ in both the diameter and relative depth of depressions. Studies demonstrate that depressions return to their resting size on completion of cell secretion $(7,8)$. No demonstrable change in pit size was detected following stimulation of secretion (7). However, enlargement of depression diameter and an increase in its relative depth after exposure to secretagogue, correlated with secretion. Additionally, exposure of pancreatic acinar cells to cytochalasin $\mathrm{B}$, a fungal toxin that inhibits actin polymerization and secretion, resulted in a $15-20 \%$ decrease in depression size and a consequent $50-60 \%$ loss of secretion (7). Results from these studies suggested depressions to be the fusion pores in pancreatic acinar cells. Furthermore, these 

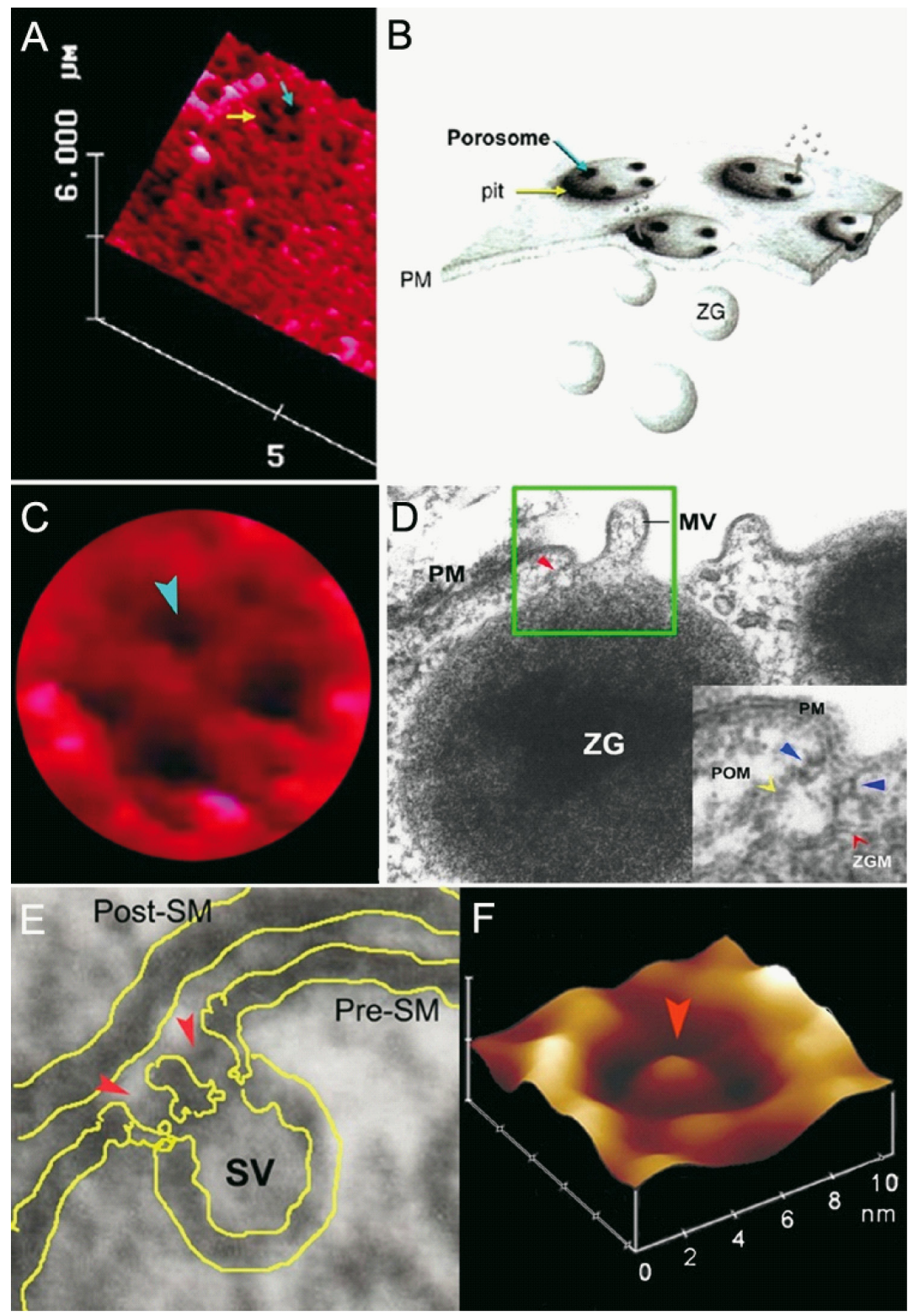

Figure 2. Porosomes or previously referred to as 'depression' at the plasma membrane in pancreatic acinar cell and at the nerve terminal. (A) AFM micrograph depicting 'pits' (yellow arrow) and 'porosomes'within (blue arrow), at the apical plasma membrane in a live pancreatic acinar cell (7). (B) To the right is a schematic drawing depicting porosomes at the cell plasma membrane (PM), where membrane-bound secretory vesicles called zymogen granules (ZG), dock and fuse to release intravesicular contents (7). (C) A high resolution AFM micrograph shows a single pit with four 100-180 nm porosomes within (7). (D) An electron micrograph depicting a porosome (red arrowhead) close to a microvilli (MV) at the apical plasma membrane (PM) of a pancreatic acinar cell (14). Note association of the porosome membrane (yellow arrowhead), and the zymogen granule membrane (ZGM) (red arrow head) of a docked ZG (inset). Cross section of a circular complex at the mouth of the porosome is seen (blue arrow head). (E) The bottom left panel shows an electron micrograph of a porosome (red arrowhead) at the nerve terminal, in association with a synaptic vesicle (SV) at the presynaptic membrane (Pre-SM). Notice a central plug at the neuronal porosome opening (10). (F) The bottom right panel is an AFM micrograph of a neuronal porosome in physiological buffer, also showing the central plug (red arrowhead) at its opening (10). It is believed that the central plug in neuronal porosomes may regulate its rapid close-open conformation during neurotransmitter release. The neuronal porosome is an order of magnitude smaller (10-15 nm) in comparison to porosome in the exocrine pancreas. 
studies demonstrated the involvement of actin in regulation of both the structure and function of depressions. Similarly, depression in resting GH cells measure $154 \pm 4.5 \mathrm{~nm}$ (mean $\pm \mathrm{SE})$ in diameter, which increases up to $40 \%(215 \pm 4.6$ $\mathrm{nm} ; \mathrm{p}<0.01)$ following exposure to a secretagogue, with no appreciable change in pit size. The enlargement of depression diameter during cell secretion and subsequent decrease accompanied by loss in secretion following exposure to actin depolymerizing agents (9), also suggested them to be the secretory portal in cells. A direct determination that depressions are the secretory portals in cells via which secretory products are expelled from the cells, was unequivocally demonstrated using immuno-AFM studies (8). Localization of gold-conjugated antibodies to various secretory proteins at these depressions finally provided the first direct evidence that secretion occur through depressions. ZGs contain the starch digesting enzyme amylase. AFM micrographs of the specific localization of gold-tagged amylase-specific antibodies at depressions following stimulation of cell secretion $(8,13)$, conclusively demonstrated depressions to be the cells secretory portal. Similarly, in somatotrophs of the pituitary gland, gold-tagged GH-specific antibodies were found to selectively localize at the depression openings following stimulation of secretion (9), which further established these sites to be the secretory portals in GH cells too. Over the years, the term "fusion pore" has been loosely referred to plasma membrane dimples that originate following a secretory stimulus, or to the continuity or channel established between opposing lipid membrane during membrane fusion. Therefore for clarity, the term "porosome" was assigned to the depression structures at the cell plasma membrane.

The porosome structure at the cytosolic compartment of the plasma membrane in the exocrine pancreas (14), and in neurons (10), has also been determined at near nm resolution in living cells. To determine the morphology of porosomes at the cytosolic compartment of pancreatic acinar cells, isolated plasma membrane preparations in near physiological buffered solution have been imaged at ultrahigh resolution using the AFM. These studies revealed scattered circular disks measuring $0.5-1 \mu \mathrm{m}$ in diameter having inverted cupshaped structures within that measure approximately $15 \mathrm{~nm}$ in height (14). On a number of occasions, ZGs ranging in size from $0.4-1 \mu \mathrm{m}$ in diameter were observed in association with one or more of the inverted cups, suggesting that the circular disks represent pits, whereas inverted cups represent porosomes, in inside-out pancreatic plasma membrane prep- arations. To further confirm that the cup-shaped structures are porosomes where secretory vesicles dock and fuse, immuno-AFM studies have been performed. Target membrane proteins, SNAP-23 (26) and syntaxin (27) (t-SNARE) and secretory vesicle-associated membrane protein (v-SNARE or VAMP) (28), are part of the conserved protein complex involved in the fusion of opposing bilayers in the presence of calcium (29-39); SNARE is an acronym derived from Soluble NSF (N-ethylmaleimide Sensitive Fusion protein) Attachment Protein Receptor. Since ZGs dock and fuse at the plasma membrane to release vesicular contents, it was hypothesized that if the inverted cups or porosomes are the secretory sites then plasma membrane-associated t-SNAREs should localize at the structure. The t-SNARE protein SNAP23 had previously been reported in pancreatic acinar cells (31). A polyclonal monospecific SNAP-23 antibody recognizing a single $23 \mathrm{kD}$ protein in immunoblots of pancreatic plasma membrane fraction, when used in immuno-AFM studies, indeed demonstrated its selective localization at the base of the cup shaped structures. These results further demonstrated that the inverted cup-shaped structures in insideout pancreatic plasma membrane preparations were indeed porosomes where secretory vesicles transiently dock and fuse to release their contents during cell secretion (13). The size and shape of the immunoisolated porosome complex has also been determined using both the negative staining EM and AFM (10-12,14) (Fig. 3).

The immunoisolated porosome complex has further been structurally and functionally reconstituted into artificial liposomes and lipid bilayer membrane (10-12,14). Transmission electron micrographs of pancreatic porosomes reconstituted into liposomes, exhibit a 150-200 nm cup-shaped basket-like morphology, similar to its native structure observed in cells and when co-isolated with ZG preparation (Fig. 2D). To test the functionality of the isolated porosome complex, purified porosomes obtained from exocrine pancreas or neurons have been reconstituted in lipid membrane of the electrophysiological bilayer setup (EPC9) and then challenged with isolated ZGs or synaptic vesicles (Fig. 4). Electrical activity of the porosome-reconstituted membrane as well as the transport of vesicular contents from the cis to the trans compartments of the bilayer chambers when monitored, further demonstrated that the lipid membrane-reconstituted porosomes, are indeed functional $(10,14)$, since in the presence of calcium, isolated secretory vesicles dock and fuse to transfer intravesicular contents from the cis to the trans compartment of the bilayer 

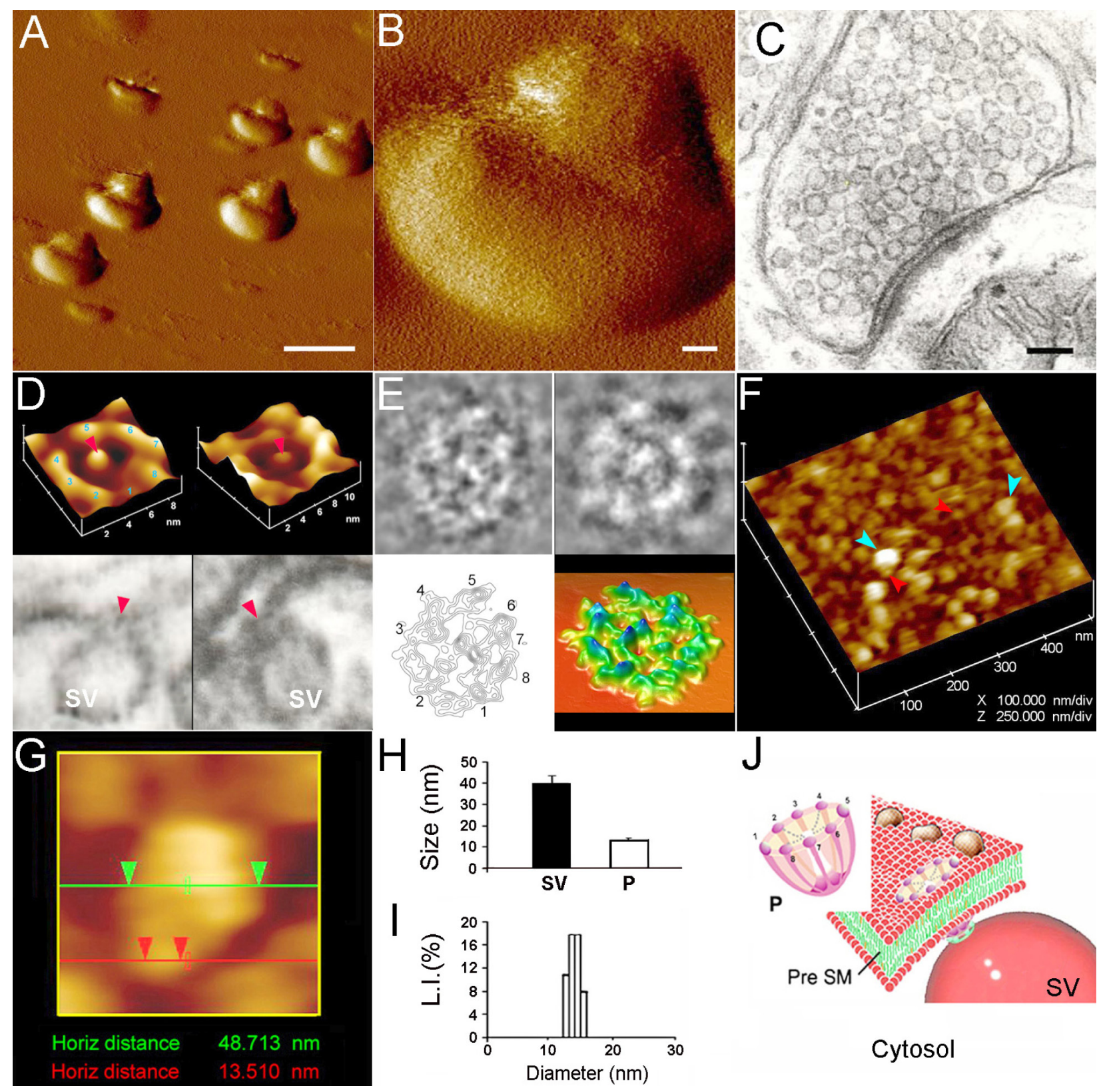

Cytosol

Figure 3. Structure and organization of the neuronal porosome complex at the nerve terminal. (A) Low resolution AFM amplitude image Bar=1mm (A) and high resolution AFM amplitude image Bar $=100 \mathrm{~nm}$ (B) of isolated rat brain synaptosomes in buffered solution. (C) Electron micrograph of a synaptosome (10), Bar $=100 \mathrm{~nm}$. (D) Structure and arrangement of the neuronal porosome complex facing the outside (Fig. D top left), and the arrangement of the reconstituted complex in PC:PS membrane (Fig. D top right). Lower panels are two transmission electron micrographs demonstrating synaptic vesicles (SV) docked at the base of cup-shaped porosome, having a central plug (red arrowhead) (12). (E) EM, electron density, and 3D contour mapping (Fig. E), provides at the nanoscale, the structure and assembly of proteins within the complex (12). (F) AFM micrograph of inside-out membrane preparations of isolated synaptosome. Note the porosomes (red arrowheads) to which synaptic vesicles are found docked (blue arrow head) (10). (G) High-resolution AFM micrograph of a synaptic vesicle docked to a porosome at the cytoplasmic compartment of the presynaptic membrane (10). (H) AFM measurements $(n=15)$ of porosomes $(P, 13.05 \pm 0.91 \mathrm{~nm})$ and synaptic vesicles $(S V, 40.15 \pm 3.14 \mathrm{~nm})$ at the cytoplasmic compartment of the presynaptic membrane (11). (I) Photon correlation spectroscopy (PCS) of immunoisolated neuronal porosome complex demonstrating a size of 12-16 nm (11). (J) Schematic illustration of a neuronal porosome at the presynaptic membrane, showing the eight ridges connected to the central plug (11).

Biomed Rev 21, 2010 


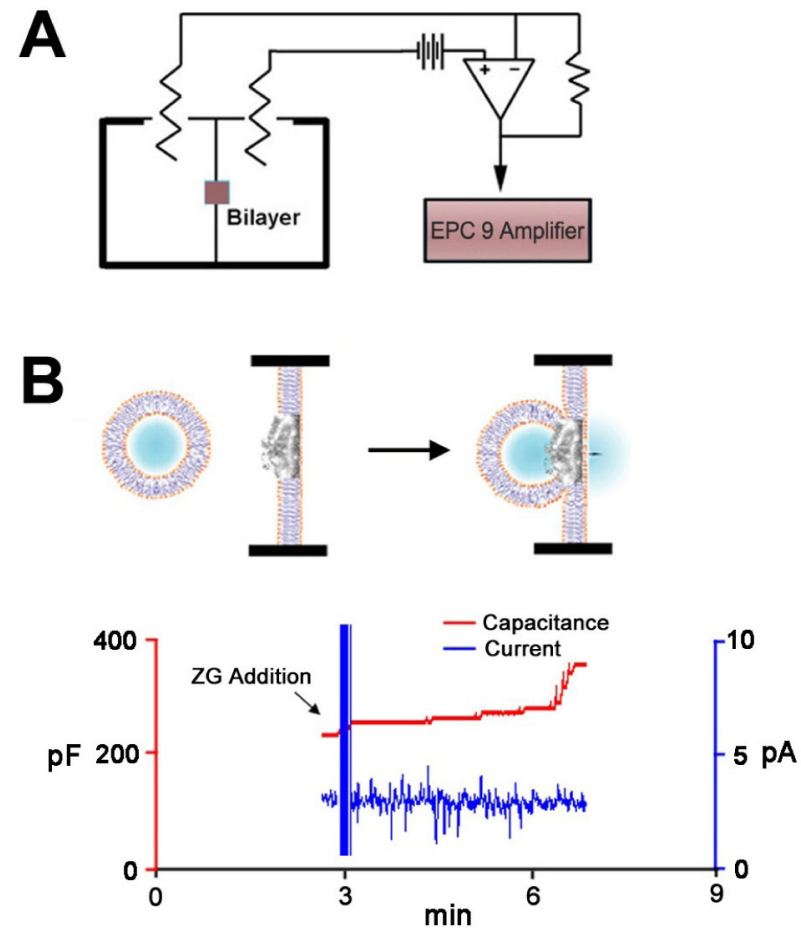

Cis Trans

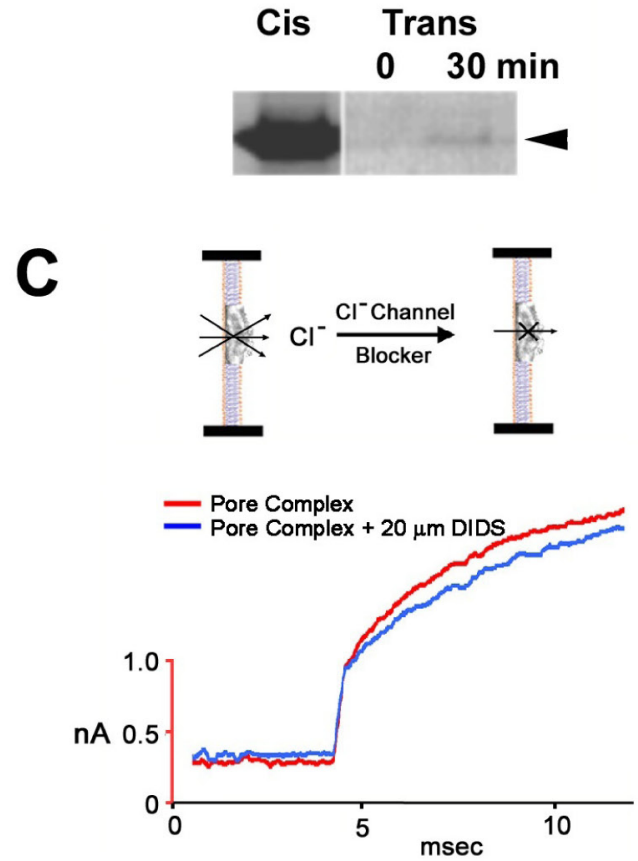

Figure 4. Lipid bilayer-reconstituted porosome complex is functional. (A) Schematic drawing of the EPC9 bilayer setup for electrophysiological measurements of porosome reconstitution and secretory vesicle fusion. (B) Zymogen granules (ZGs) added to the cis side of the bilayer fuse with the reconstituted porosomes, as demonstrated by an increase in capacitance and current activities, and a concomitant time dependent release of amylase (a major ZG content) to the trans side of the membrane. The movement of amylase from the cis to the trans side of the chamber was determined by immunoblot analysis of the contents in the cis and the trans chamber over time. (C) As demonstrated by immunoblot analysis of the immunoisolated complex, electrical measurements in the presence and absence of chloride ion channel blocker DIDS, demonstrate the presence of chloride channels in association with the complex (14). 
chamber. ZGs fused with the porosome-reconstituted bilayer as demonstrated by an increase in capacitance and conductance, and a time-dependent transport of the ZG enzyme amylase from cis to the trans compartment of the bilayer chamber. Amylase was detected using immunoblot analysis of the buffer in the cis and trans compartments of the bilayer chambers. Furthermore, chloride channel activity present in the reconstituted porosome complex was found to be critical for the porosome function as the chloride channel blocker DIDS inhibited the porosome function.

Similarly, the structure and biochemical composition of the neuronal porosome, and the docking and fusion of synaptic vesicles at the neuronal porosome complex has also been demonstrated. AFM, EM, and electron density measurements followed by contour mapping, and 3D topography of the neuronal porosome, have further provided an understanding of the arrangement of proteins at $\mathrm{nm}$ resolution within this complex (12). Results from these studies demonstrate that proteins at the central plug of the porosome interact with proteins at the periphery of the complex conforming to its eight-fold symmetry (Fig. 3D,E). Furthermore, at the center of the porosome complex representing the porosome base where synaptic vesicles dock and transiently fuse, SNARE proteins assemble in a ring conformation (40). The SNARE ring was found to be composed of merely three SNARE pairs having a $1-1.5 \mathrm{~nm}$ in diameter channel for the express release of neurotransmitters from synaptic vesicles via the porosome to the synaptic cleft (40). These studies demonstrate that porosomes are permanent structures at the presynaptic membrane of nerve terminals where synaptic vesicles transiently dock and fuse to release neurotransmitters. Photon correlation spectroscopy (PCS) of isolated porosome complexes further confirmed that neuronal porosomes measure on average $14 \mathrm{~nm}$ (Fig. 3I). In PCS measurements, the size distribution of isolated porosome complexes is obtained from plots of the relative intensity of light scattered by particles of known sizes and then their correlation function is calculated (41). Negative staining EM performed using low electron dose, in a Tacnai 20 electron microscope operating at $200 \mathrm{kV}$ (42), further demonstrated that proteins at the central plug of the porosome complex interact with proteins at the periphery of the structure (12). Similar to AFM micrographs, approximately 8 interconnected protein densities were observed at the lip of the porosome complex in electron micrographs. These densities were also connected to the central plug via spoke-like structures. Electron density and contour maps and resultant 3D topology profiles of the porosome complex provided further details of the circular arrangement of proteins and their connection to the central plug via distinct spokes (Fig. 3E). The contour map of proteins within the neuronal porosome complex, have been determined using previously published procedures (43-45). These studies provide the arrangement of proteins at the nm scale within the neuronal porosome complex. The next level of understanding of this supramolecular structure would require electron diffraction studies on isolation complexes that are currently under way in our laboratory.

In summary, these studies demonstrate porosomes to be permanent supramolecular lipoprotein structures at the cell plasma membrane, where membrane-bound secretory vesicles transiently dock and fuse to release intravesicular contents to the outside. Porosomes are therefore the universal secretory machinery of the cell $(24,25)$.

\section{Fusion of secretory vesicles at the base of the porosome complex}

As previously outlined, membrane fusion is mediated via a specialized set of proteins present in opposing bilayers $(26,32,34,35)$. Target membrane proteins, SNAP-25 and syntaxin (t-SNAREs) and secretory vesicle-associated protein ( $\mathrm{v}$-SNARE), are part of the conserved protein complex involved in the fusion of opposing lipid membranes. The structure and arrangement of membrane-associated full length SNARE complex was first determined using AFM (28). This study demonstrated that t-SNAREs and v-SNARE, when present in opposing bilayers, interact in a circular array to form supramolecular ring complexes each measuring a few nanometers. The size of the ring complex is directly proportional to the curvature of the opposing bilayers (33). In the presence of calcium, the ring-complex helps in establishing continuity between the opposing bilayers $(34,35,38)$. In contrast, soluble v-SNARE and t-SNAREs neither assemble any specific and organized pattern nor form such conducting channels in the absence of membranes. Once v-SNARE and t-SNAREs residing in opposing bilayers meet, the resulting SNARE complex overcome the repulsive forces between opposing bilayers, bringing them closer to within a distance of 2.8-3 $\AA$ that allows calcium bridging of the opposing phospholipids head groups leading to local dehydration and membrane fusion $(34,35,38)$. VAMP and syntaxin are both integral membrane proteins, with the soluble SNAP-25 associating with syntaxin. Hence, the key to our understanding of 
SNARE-induced membrane fusion necessitates determination of the atomic arrangement, and of the interaction that occurs during complex formation between membrane-associated v-SNARE and t-SNAREs. Ideally, x-ray crystallography would help elucidate the chemistry of SNARE-induced membrane fusion in cells. So far the structural details of membrane-associated $\mathrm{t}-\mathrm{v}$-SNARE complex at the atomic level have eluded us, primarily due to the solubility issues of membrane-associated SNAREs. This is further compounded by the fact that $\mathrm{v}$-SNARE and t-SNAREs need to reside in opposing membranes when they meet in order to assemble in a physiologically relevant SNARE complex. The remaining option of using nuclear magnetic resonance (NMR) spectroscopy too has been of little help, since the size of the $\mathrm{t}-\mathrm{v}$ SNARE ring complexes is beyond the maximum limit for NMR studies (>100 kD). Regardless of these limitations, AFM spectroscopy has provided for the first time an understanding of the structure, assembly, and disassembly of membrane-associated t-/v-SNARE complexes in physiological buffer solution at $\mathrm{nm}$ to sub $\mathrm{nm}$ resolution (32-40). Combined with AFM imaging, the bilayer electrophysiological setup (EPC9) has enabled structure-function studies during fusion of v-SNARE-reconstituted liposomes with t-SNAREreconstituted membrane, and vice-versa. Results from these studies have demonstrated that t-SNAREs and v-SNARE when present in opposing membrane interact and assemble in a circular array, and in the presence of calcium form conducting channels (32). Addition of purified recombinant $\mathrm{v}$ SNARE to a t-SNARE-reconstituted lipid membrane results in non-physiological interactions without influencing the electrical properties of the membrane (32). However in the presence of calcium, when v-SNARE vesicles are added to t-SNARE reconstituted membrane or vice-versa, SNAREs assemble in a ring conformation. The resultant increase in membrane capacitance and conductance demonstrate the establishment of continuity between the opposing t-SNARE and $\mathrm{v}$-SNARE reconstituted bilayers. These results confirm that $\mathrm{t}-\mathrm{v}$-SNAREs are required to reside in opposing membrane, as they exist in the physiological state in cells, to allow appropriate interactions leading to membrane fusion in the presence of calcium. Studies using SNARE-reconstituted liposomes and bilayers $(34,35)$ further demonstrated: $(i)$ a low fusion rate ( $\mathrm{t}=16 \mathrm{~min}$ ) between $\mathrm{t}-\mathrm{SNARE}$ and v-SNARE-reconstituted liposomes in the absence of $\mathrm{Ca}^{2+}$ and (ii) whereas exposure of $\mathrm{t}-/ \mathrm{v}$-SNARE liposomes to $\mathrm{Ca}^{2+}$ drives vesicle fusion on a near physiological relevant time-scale $(\mathrm{t} \sim 10 \mathrm{~s})$, demonstrating that $\mathrm{Ca}^{2+}$ and SNAREs in combination are the minimal fusion machinery of cells $(34,35)$. Native and synthetic vesicles exhibit a significant negative surface charge primarily due to the polar phosphate head groups, generating a repulsive force that prevent the aggregation and fusion of opposing vesicles. In cells, SNAREs provide direction and specificity, bringing opposing bilayers closer to within a distance of 2-3 $\AA(34,35)$ enabling $\mathrm{Ca}^{2+}$ bridging and membrane fusion. The bound $\mathrm{Ca}^{2+}$ then leads to the expulsion of water between the bilayers at the bridging site leading to lipid mixing and membrane fusion. Hence SNAREs, besides bringing opposing bilayers closer, dictate the site and size of the fusion area during cell secretion. The size of the t-/v-SNARE complex is dictated by the curvature of the opposing membranes (33), hence the smaller the vesicle, the smaller is the channel formed. A unique set of chemical and physical properties of the $\mathrm{Ca}^{2+}$ ion makes it ideal for participating in the membrane fusion reaction. Calcium ion exists in its hydrated state within the cell. The properties of hydrated calcium have been extensively studied using $\mathrm{x}$-ray diffraction, neutron scattering, in combination with molecular dynamics simulations (46-49). The molecular dynamic simulations include three-body corrections compared with ab initio quantum mechanics/molecular mechanics molecular dynamics simulations. First principle molecular dynamics has also been used to investigate the structural, vibrational, and energetic properties of $\left[\mathrm{Ca}\left(\mathrm{H}_{2} \mathrm{O}\right)_{n}\right]^{2+}$ clusters, and the hydration shell of the calcium ion (48). These studies demonstrate that hydrated calcium $\left[\mathrm{Ca}\left(\mathrm{H}_{2} \mathrm{O}\right)_{n}\right]^{2+}$ has more than one shell around the $\mathrm{Ca}^{2+}$, with the first hydration shell having six water molecules in an octahedral arrangement (24). In studies using light scattering and $\mathrm{x}$-ray diffraction of SNARE-reconstituted liposomes, it has been demonstrated that fusion proceeds only when $\mathrm{Ca}^{2+}$ ions are available between the $\mathrm{t}$ - and $\mathrm{v}$ SNARE-apposed proteoliposomes $(34,35)$. Mixing of t- and v-SNARE proteoliposomes in the absence of $\mathrm{Ca}^{2+}$ leads to a diffuse and asymmetric diffractogram in X-ray diffraction studies, a typical characteristic of short range ordering in a liquid system (47). In contrast, when t-SNARE and vSNARE proteoliposomes are mixed in the presence of $\mathrm{Ca}^{2+}$, it leads to a more structured diffractogram with approximately a $12 \%$ increase in $\mathrm{x}$-ray scattering intensity, suggesting an increase in the number of contacts between opposing bilayers established presumably through calcium-phosphate bridges, as previously suggested $(34,35,46)$. The ordering effect of $\mathrm{Ca}^{2+}$ on inter-bilayer contacts observed in x-ray studies (29) 
is in good agreement with light and AFM spectroscopic studies, suggesting a close apposition of PO-lipid head groups in the presence of $\mathrm{Ca}^{2+}$, followed by formation of $\mathrm{Ca}^{2+}-\mathrm{PO}$ bridges between the adjacent bilayers $(34,35,50)$. X-ray diffraction studies show that the effect of $\mathrm{Ca}^{2+}$ on bilayers orientation and inter-bilayer contacts is most prominent in the area of $3 \AA$, with additional appearance of a new peak at position $2.8 \AA$, both of which are within the ionic radius of $\mathrm{Ca}^{2+}(35)$. These studies further suggest that the ionic radius of $\mathrm{Ca}^{2+}$ makes it an ideal player in the membrane fusion reaction. Hydrated calcium $\left[\mathrm{Ca}\left(\mathrm{H}_{2} \mathrm{O}\right)_{n}\right]^{2+}$ however, with a hydration shell having six water molecules and measuring $\sim 6 \AA$ would be excluded from the $\mathrm{t}-\mathrm{v}$-SNARE apposed inter-bilayer space, hence calcium has to be present in the buffer solution when t-SNARE vesicles and v-SNARE vesicles meet. Indeed, studies demonstrate that if t-SNARE and v-SNARE vesicles are allowed to mix in a calcium-free buffer, there is no fusion following post addition of calcium (34). How does calcium work? Calcium bridging of apposing bilayers may lead to the release of water from the hydrated $\mathrm{Ca}^{2+}$ ion leading to bilayer destabilization and membrane fusion. Additionally, the binding of calcium to the phosphate head groups of the apposing bilayers may also displace the loosely coordinated water at the PO-lipid head groups, resulting in further dehydration, leading to destabilization of the lipid bilayer and membrane fusion. Recent studies from our laboratory (38), using molecular dynamics simulations in the isobaricisothermal ensemble to determine whether $\mathrm{Ca}^{2+}$ was capable of bridging opposing phospholipid head groups in the early stages of the membrane fusion process, demonstrate indeed this to be the case. Furthermore, the distance between the oxygen atoms of the opposing PO-lipid head groups bridged by calcium is in agreement with the $2.8 \AA$ distance previously determined using X-ray diffraction measurements. The hypothesis that there is loss of coordinated water both from the hydrated calcium ion and oxygen of the phospholipid head groups in opposing bilayers following calcium bridging, is further demonstrated in our study.

In the presence of ATP, the highly stable, membranedirected, and self-assembled t-/v-SNARE complex, can be disassembled by a soluble ATPase, the $N$-ethylmaleimidesensitive factor (NSF). Careful examination of the partially disassembled t-/v-SNARE bundles within the complex using AFM demonstrates a left-handed super coiling of SNAREs. These results demonstrate that $\mathrm{t}-\mathrm{v}$-SNARE disassembly requires the right-handed uncoiling of each SNARE bundle within the ring complex, demonstrating NSF to behave as a right-handed molecular motor (37). Furthermore, using circular dichroism (CD) spectroscopy, we reported for the first time that both t-SNAREs and v-SNARE and their complexes in buffered suspension exhibit defined peaks at CD signals of 208 and $222 \mathrm{~nm}$ wavelengths, consistent with a higher degree of helical secondary structure (39). Surprisingly, when incorporated in lipid membrane, both SNAREs and their complexes exhibit reduced folding. NSF, in presence of ATP, disassembles the SNARE complex as reflected from the CD signals demonstrating elimination of $\alpha$-helices within the structure. These results further demonstrate that NSF-ATP is sufficient for the disassembly of the t-/v-SNARE complex, providing a molecular understanding of SNARE-induced membrane fusion in cells.

\section{Expulsion of intravesicular contents to the outside via the porosome}

As previously outlined, isolated live pancreatic acinar cells in near physiological buffer when imaged using AFM at high force $(200-300 \mathrm{pN})$, demonstrate the size and shape of the ZGs lying immediately below the apical plasma membrane of the cell (Fig. 1). Within $2.5 \mathrm{~min}$ of exposure to a physiological secretory stimulus, the majority of ZGs within cells swell (Fig. 1) followed by a decrease in ZG size by which time secretion is complete. These studies reveal for the first time in living cells, intracellular swelling of secretory vesicles following stimulation of cell secretion and their deflation following partial discharge of vesicular contents (6). No loss of secretory vesicles is observed throughout the experiment. Measurements of intracellular ZG size further reveal that different vesicles swell differently, following a secretory stimulus. For example, the ZG marked by the red arrowhead swelled to show a $23-25 \%$ increase in diameter, in contrast to the green arrowhead-marked $\mathrm{ZG}$, which increased by only $10-11 \%$ (Fig. 1). This differential swelling among ZGs within the same cell may explain why following stimulation of secretion, some intracellular ZGs demonstrate the presence of less vesicular content than others, and hence have discharged more of their contents. To determine precisely the role of swelling in vesicle-plasma membrane fusion and in the expulsion of intravesicular contents, an electrophysiological ZG-reconstituted lipid bilayer fusion assay has been employed. The ZGs used in the bilayer fusion assays were characterized for their purity and their ability to respond to a swelling stimulus. As previously reported $(51,52)$, expo- 
sure of isolated ZGs to GTP results in ZG swelling. Similar to what is observed in living acinar cells, each isolated ZG responds differently to the same swelling stimulus. This differential response of isolated ZGs to GTP has been further assessed by measuring changes in the volume of isolated ZGs of different sizes (6). ZGs in the exocrine pancreas range in size from 0.2 to $1.3 \mu \mathrm{m}$ in diameter (51), and not all ZGs are found to swell following a GTP challenge (6). In most ZGs, volume increases are between 5-20\%, however larger increases of up to $45 \%$ are observed only in vesicles ranging from $250 \mathrm{~nm}$ to $750 \mathrm{~nm}$ in diameter. In the electrophysiological bilayer fusion assay, immunoisolated porosome complex from the exocrine pancreas, are functionally reconstituted (14) into the lipid membrane of the bilayer apparatus where membrane conductance and capacitance can be continually monitored (Fig. 5A) (6). Reconstitution of the porosome into the lipid membrane results in a small increase in capacitance (Fig. 5B), possibly due to the increase in membrane surface area. Isolated ZGs when added to the cis compartment of the bilayer chamber, fuse at the porosome-reconstituted lipid membrane (Fig. 5A) and is detected as a step increase in membrane capacitance (Fig. 5B). Even after $15 \mathrm{~min}$ of ZG addition to the cis compartment of the bilayer chamber, little or no release of the intra-vesicular enzyme a-amylase is detected in the trans compartment of the bilayer chamber (Fig. 5C, 5D). On the contrary, exposure of ZGs to $20 \mathrm{mM} \mathrm{GTP \text {, }}$ induced swelling and resulted both in the potentiation of fusion as well as a robust expulsion of a-amylase into the trans compartment of the bilayer chamber observed in immunoblot assays (Fig. 5C, 5D). These studies demonstrate that during cell secretion, secretory vesicle swelling is required for the efficient expulsion of intravesicular contents. This mechanism of vesicular expulsion during cell secretion may explain why partially empty vesicles are generated in cells following secretion. The presence of empty secretory vesicles could result from multiple rounds of fusion-swelling-expulsion cycles a vesicle may undergo during the secretory process, reflecting on the precise and regulated nature of cell secretion.

\section{CONCLUSION}

This article reviews our current understanding of the molecular machinery and mechanism involved in cell secretion. Porosomes are specialized plasma membrane structures universally present in secretory cells, from exocrine and endocrine cells to neuroendocrine cells and neurons. Since porosomes in exocrine and neuroendocrine cells measure 100-180 nm, and only a $20-35 \%$ increase in porosome diameter is demonstrated following the docking and fusion of secretory vesicles ranging in diameter of $0.2-1.2 \mu \mathrm{m}$, it is concluded that secretory vesicles "transiently" dock and fuse at the base of the porosome complex to release their contents to the outside. Furthermore, isolated live cells in near physiological buffer when imaged using AFM, demonstrate the size and shape of the secretory vesicles lying immediately below the apical plasma membrane of the cell. Following exposure to a secretory stimulus secretory vesicles swell followed by a decrease in vesicle size. No loss of secretory vesicles is observed following secretion, demonstrating transient fusion and partial discharge of vesicular contents during cell secretion. In agreement, a studies demonstrate that "secretory granules are recaptured largely intact after stimulated exocytosis in cultured endocrine cells" (16), "single synaptic vesicles fusing transiently and successively without loss of identity" (17), and "zymogen granule exocytosis is characterized by long fusion pore openings and preservation of vesicle lipid identity" (18). This is in contrast to the general belief that in mammalian cells, secretory vesicles completely merge at the cell plasma membrane resulting in passive diffusion of vesicular contents to the cell exterior, and the consequent retrieval of excess membrane by endocytosis at a later time. Additionally, a major logistical problem with complete merger of secretory vesicle membrane at the cell plasma membrane is the generation of partially empty vesicles following cell secretion. It is fascinating how even single-cell organisms have developed such specialized secretory machinery, like the secretion apparatus of Toxoplasma gondii, the contractile vacuole in paramecium, and the secretory structures in bacteria. Hence it comes as no surprise that mammalian cells have evolved such highly specialized and sophisticated structures - the porosome complex for cell secretion. The discovery of the porosome, and an understanding of its structure and dynamics at $\mathrm{nm}$ resolution and in real time in living cells, its composition, and its functional reconstitution in lipid membrane, and the molecular mechanisms of SNARE-induced membrane fusion have greatly advanced our understanding of cell secretion. It is evident that the secretory process in cells is a well coordinated, highly regulated and a finely tuned biomolecular orchestra. Clearly, these findings could not have advanced without the AFM, and therefore this powerful tool has greatly contributed to a new understanding of the cell. The AFM has enabled the determination of live cellular structure-function at $\mathrm{nm}$ to $\AA$ resolution, in real time, contributing to the birth 
Figure 5. Fusion of isolated ZGs at porosome-reconstituted bilayer and GTP-induced expulsion of aamylase. (A) Schematic diagram of the EPC9 bilayer apparatus showing the cis and trans chambers. Isolated ZGs when added to the cis chamber, fuse at the bilayersreconstituted porosome. Addition of GTP to the cis chamber induces $Z G$ swelling and expulsion of its contents such as a-amylase to the trans bilayers chamber. (B) Capacitance traces of the lipid bilayer from three separate experiments following reconstitution of porosomes (green arrowhead), addition of ZGs to the cis chamber (blue arrowhead), and the red arrowhead represents the $5 \mathrm{~min}$ time point after ZG addition. Note the small increase in membrane capacitance following porosome reconstitution, and a greater increase when ZGs fuse at the bilayers. (C) In a separate experiment, 15 min after addition of ZGs to the cis chamber, 20 mM GTP was introduced. Note the increase in capacitance, demonstrating potentiation of $Z G$ fusion. Flickers in current trace represent current activity. (D) Immunoblot analysis of a-amylase in the trans chamber fluid at different times following exposure to ZGs and GTP. Note the undetectable levels of aamylase even up to 15 min following $Z G$ fusion at the bilayer. However, following exposure to GTP, significant amounts of a-amylase from within ZGs were expelled into the trans bilayers chamber (6).
A
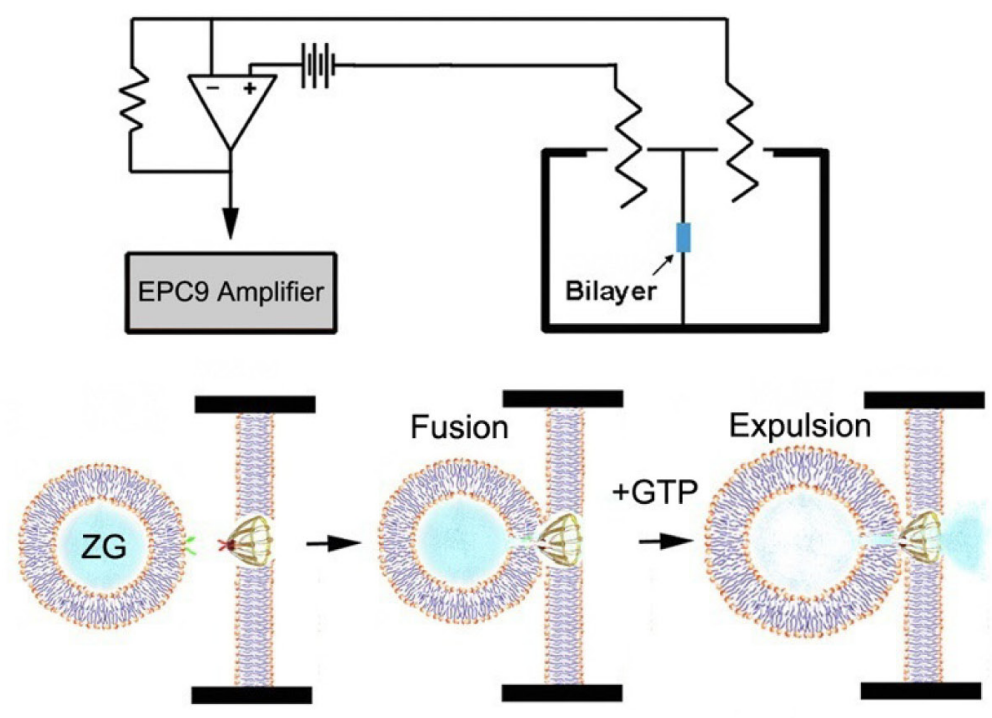

B

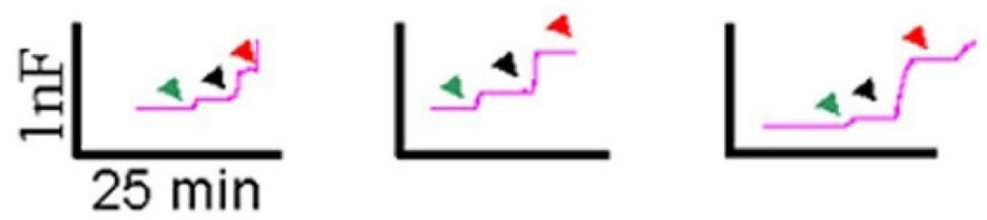

C

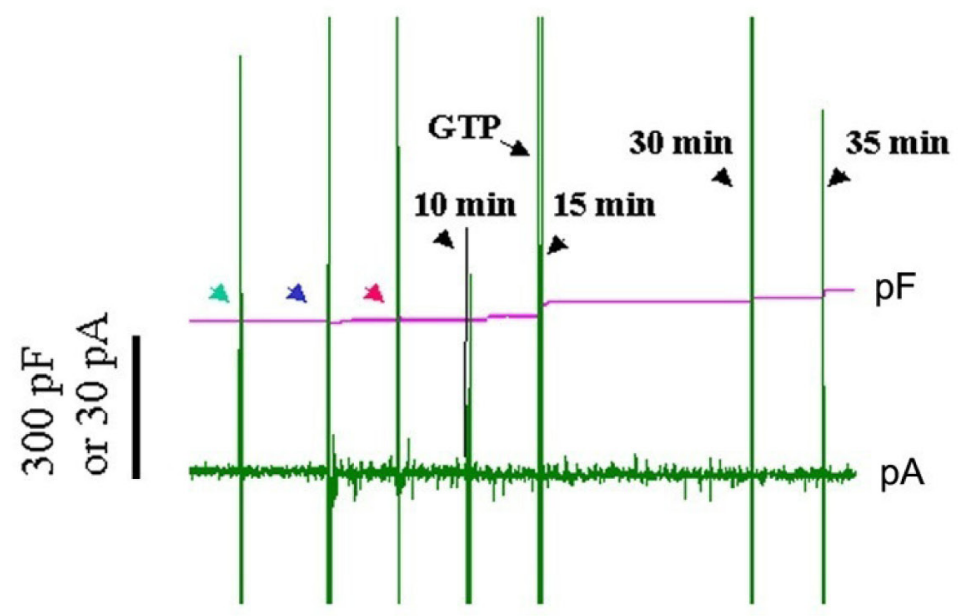

D

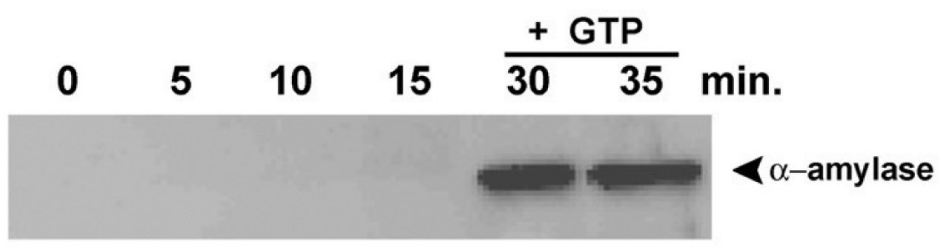


of the new field of NanoCellBiology. Future directions will involve an understanding of the protein distribution and their arrangement at atomic resolution in the porosome complex, and a similar understanding of the structure of the $\mathrm{t}-\mathrm{v}$ SNARE ring complex. Determination of the atomic structure of membrane-associated full-length SNAREs and their complexes as well as the neuronal porosome complex are being carried out using electron crystallography in our laboratory. Indeed, the nanobiology of porosomes became one of the forefronts of cell research today $(53,54)$.

\section{ACKNOWLEDGEMENTS}

The author thanks the many students and collaborators who have participated in the various studies discussed in this article. The author thanks Won Jin Cho for help in formatting the manuscript. Research in the author's laboratory was support by Grants from the NIH, NSF, and Wayne State University.

\section{REFERENCES}

1. Katz B. The transmission of impulses from nerve to muscle and the subcellular unit of synaptic action. Proc Roy Soc B 1962;155:455-479.

2. Folkow B, Häggendal J, Lisander B. Extent of release and elimination of noradrenalin at peripheral adrenergic nerve terminal. Acta Physiol Scand Suppl 1967;307:138.

3. Folkow B, Häggendal J. Some aspects of the quantal release of the adrenergic transmitter. Springer-Verlag Bayer Symp 1970;II:91-97.

4. Folkow B. Transmitter release at the adrenergic nerve endings: total exocytosis or fractional release? News Physiol Sci 1997;12:32-35.

5. Neher E. Secretion without full fusion. Nature 1993;363:497-498.

6. Kelly M, Cho WJ, Jeremic A, Abu-Hamdah R, Jena BP. Vesicle swelling regulates content expulsion during secretion. Cell Biol Int 2004;28:709-16.

7. Schneider SW, Sritharan KC, Geibel JP, Oberleithner H, Jena BP. Surface dynamics in living acinar cells imaged by atomic force microscopy: Identification of plasma membrane structures involved in exocytosis. Proc Natl Acad Sci USA 1997;94:316-21.

8. Cho S-J, Quinn AS, Stromer MH, Dash S, Cho J, Taatjes DJ, Jena BP. Structure and dynamics of the fusion pore in live cells. Cell Biol Int 2002;26:35-42.

9. Cho S-J, Jeftinija K, Glavaski A, Jeftinija S, Jena BP,
Anderson LL. Structure and dynamics of the fusion pores in live GH-secreting cells revealed using atomic force microscopy. Endocrinology 2002;143:1144-8.

10. Cho WJ, Jeremic A, Rognlien KT, Zhvania MG, Lazrishvili I, Tamar B, Jena BP. Structure, isolation, composition and reconstitution of the neuronal fusion pore. Cell Biol Int 2004;28:699-708.

11. Cho WJ, Jeremic A, Jin H, Ren G, Jena BP. Neuronal fusion pore assembly requires membrane cholesterol. Cell Biol Int 2007;31:1301-1308.

12. Cho WJ, Ren G, Jena BP. EM 3D contour maps provide protein assembly at the nanoscale within the neuronal porosome complex. J Microscopy 2008;232:106-111.

13. Jena BP, Cho S-J, Jeremic A, Stromer MH, Abu-Hamdah R. Structure and composition of the fusion pore. Biophys J 2003;84:1-7.

14. Jeremic A, Kelly M, Cho S-J, Stromer MH, Jena BP. Reconstituted Fusion Pore. Biophys J 2003;85:2035-2043.

15. Siksou L, Rostaing P, Lechaire JP, Boudier T, Ohtsuka T, Fejtova A, et al. Three-dimensional architecture of presynaptic terminal cytomatrix. $J$ Neurosci 2007;27:68686877.

16. Taraska JW, Perrais D, Ohara-Imaizumi M, Nagamatsu S, Almers W. Secretory granules are recaptured largely intact after stimulated exocytosis in cultured endocrine cells. Proc Natl Acad Sci USA 2003;100;2070-2075.

17. Aravanis AM, Pyle JL, Tsien RW. Single synaptic vesicles fusing transiently and successively without loss of identity. Nature 2003;423:643-647.

18. Thorn P, Fogarty KE, Parker I. Zymogen granule exocytosis is characterized by long fusion pore openings and preservation of vesicle lipid identity. Proc Natl Acad Sci USA 2004;101:6774-6779.

19. Joiner KA, Ross DS. Secretory traffic in the eukaryotic parasite Toxoplasma gondii: less is more. $J$ Cell Biol 2002;157:557-563.

20. Hausmann K, Allen RD. Membranes and microtubules of the excretory apparatus of Paramecium caudatum. $\mathrm{Cy}$ tobiologie 1997;15:303-320.

21. Kubori T, Matsushima Y, Nakamura D, Uralil J, LaraTejero M, Sukhan A, et al. Supramolecular structure of the Salmonella typhimurium type III protein secretion system. Science 1998;280:602-605.

22. Cho S-J, Wakade A, Pappas GD, Jena BP. New structure involved in transient membrane fusion and exocytosis. Ann New York Acad Sci 2002;971:254-256. 
23. Jena BP. Discovery of the porosome: revealing the molecular mechanism of secretion and membrane fusion in cells. J Cellular Mol Med 2004;8: 1-21.

24. Jena BP. Porosome: the universal molecular machinery for cell secretion. Mol Cells 2008;26:517-529.

25. Jena BP. Porosome: the secretory portal in cells. Biochemistry 2009;49:4009-4018.

26. Oyler GA, Higgins GA, Hart RA, Battenberg E, Billingsley $\mathrm{M}$, Bloom FE, et al. The identification of a novel synaptosomal-associated protein, SNAP-25, differentially expressed by neuronal subpopulations. J Cell Biol 1989;109:3039-52.

27. Bennett MK, Calakos N, Schller RH. Syntaxin: A synaptic protein implicated in docking of synaptic vesicles at presynaptic active zones. Science 1992;257: 255259.

28. Trimble WS, Cowan DW, Scheller RH. VAMP-1: A synaptic vesicle-associated integral membrane protein. Proc Natl Acad Sci USA 1988;85: 4538-4542.

29. Malhotra V, Orci L, Glick BS, Block MR, Rothman JE. Role of an N-ethylmaleimide-sensitive transport component in promoting fusion of transport vesicles with cisternae of the Golgi stack. Cell 1988;54: 221227.

30. Wilson DW, Whiteheart SW, Wiedmann M, Brunner M, Rothman JE. A multisubunit particle implicated in membrane fusion. J Cell Biol 1992;117: 531-538.

31. Gaisano HY, Sheu L, Wong PP, Klip A, Trimble WS. SNAP-23 is located in the basolateral plasma membrane of rat pancreatic acinar cells. FEBS Lett 1997;414: 298302.

32. Cho S-J, Kelly M, Rognlien KT, Cho JA, Hörber JK, Jena BP. SNAREs in opposing bilayers interact in a circular array to form conducting pores. Biophys $J$ 2002;83:2522-2527.

33. Cho WJ, Jeremic A, Jena BP. Size of supramolecular SNARE complex: Membrane-directed self-assembly. $J$ Am Chem Soc 2005;127:10156-10157.

34. Jeremic A, Kelly M, Cho JA, Cho S-J, Hörber JK, Jena BP. Calcium drives fusion of SNARE-apposed bilayers. Cell Biol Int 2004;28:19-31.

35. Jeremic A, Cho WJ, Jena BP. Membrane fusion: what may transpire at the atomic level. J Biol Phys Chem 2004;4:139-142.

36. Jeremic A, Quinn AS, Cho WJ, Taatjes DJ, Jena BP. Energy-dependent disassembly of self-assembled SNARE com- plex: Observation at nanometer resolution using atomic force microscopy. J Am Chem Soc 2006;128:26-27.

37. Cho WJ, Jena BP. N-ethymaleimide sensitive factor is a right-handed molecular motor. $J$ Biomed Nanotech 2007;3:209-211.

38. Potoff JJ, Issa Z, Manke Jr CW, Jena BP. $\mathrm{Ca}^{2+}$-dimethylphosphate complex formation: providing insight into $\mathrm{Ca}^{2+}$ mediated local dehydration and membrane fusion in cells. Cell Biol Int 2008;32:361-366.

39. Cook JD, Cho WJ, Stemmler TL, Jena BP. Circular dichroism (CD) spectroscopy of the assembly and disassembly of SNAREs: the proteins involved in membrane fusion in cells. Chem Phys Lett 2008;462:6-9.

40. Cho W-J, Shin L, Ren G, Jena BP. Structure of membrane-associated neuronal SNARE complex: Implication in neurotransmitter release. $\mathrm{J}$ Cell $\mathrm{Mol}$ Med 2009;13:4161-4165.

41. Jeremic A, Cho WJ, Jena BP. Involvement of water channels in synaptic vesicle swelling. Exp Biol Med 2005;230:674-680.

42. Ludtke SJ, Baldwin PR, Chiu W. EMAN: semiautomated software for high-resolution single-particle reconstructions. J Struct Biol 1999;128: 82-97.

43. Frank J, Radermacher M, Penczek P, Zhu J, Li Y, Lasjadj $\mathrm{M}$, et al. SPIDER and WEB: processing and visualization of images in 3D electron microscopy and related fields. J Struct Biol 1996;116:190-199.

44. Goddard TD, Huang C. C, Ferrin TE. Software extensions to UCSF Chimera for interactive visualization of large molecular assemblies. Structure 2005;13: 473-482.

45. Pettersen EF, Goddard TD, Huang CC, Couch GS, Greenblatt DM, Meng EC, et al. UCSF Chimera - A visualization system for exploratory research and analysis. J Comput Chem 2004;25:1605-1612.

46. Portis A, Newton C, Pangborn W, Papahadjopoulos D. Studies on the mechanism of membrane fusion: evidence for an intermembrane $\mathrm{Ca}^{2+}$ phospholipid complex, synergism with $\mathrm{Mg}^{2+}$, and inhibition by spectrin. Biochemistry 1979;18,780-790.

47. McIntosh TJ. Short-range interactions between lipid bilayers measured by X-ray diffraction. Curr Opin Struct Biol 2000;10:481-485.

48. Bako I, Hutter J, Palinkas G. Car-Parrinello molecular dynamics simulation of the hydrated calcium ion. $J$ Chem Phys 2002;117:9838-9843.

49. Chialvo AA, Simonson JM. The structure of $\mathrm{CaCl}_{2}$ aque- 
ous solutions over a wide range of concentration. Interpretation of diffraction experiments via molecular simulation. J Chem Phys 2003;19:8052-8061.

50. Laroche G, Dufourc EJ, Dufoureq J, Pezolet M. Structure and dynamics of dimyristoylphosphatidic acid/ calcium complex by $2 \mathrm{H}$ NMR, infrared, spectroscopies and small-angle $\mathrm{x}$-ray diffraction. Biochemistry 1991;30:3105-4114.

51. Jena BP, Schneider SW, Geibel JP, Webster P, Oberleithner H, Sritharan KC. $\mathrm{G}_{\mathrm{i}}$ regulation of secretory vesicle swelling examined by atomic force microscopy. Proc
Natl Acad Sci USA 1997;94:13317-13322.

52. Cho S-J, Sattar AK, Jeong EH, Satchi M, Cho J, Dash S, et al. Aquaporin 1 regulates GTP-induced rapid gating of water in secretory vesicles. Proc Natl Acad Sci USA 2002;99:4720-4724.

53. Wheatley DN. Pores for thought: further landmarks in the elucidation of the mechanisms of secretion. Cell Biol Int 2007; 31: 1297-1300.

54. Wheatley DN. Another decade of evidences in research on primary cilia, porosomes and neosis: some passing thoughts at 70. Cell Biol Int 2010; 34: 335-337. 